\title{
Results of Ponseti Casting for Clubfoot in a Tertiary Public Hospital
}

\author{
Ilian Dominiq D. Eusebio, MD* and Carlo Emmanuel J. Sumpaico, MD* \\ Department of Orthopedics, Philippine General Hospital, University of the Philippines Manila
}

\begin{abstract}
Introduction. Congenital talipes equinovarus (CTEV), also called clubfoot, is one of the most common orthopedic congenital anomalies. However, there is no formal study of the condition here in the Philippines, and data is sparse regarding the epidemiology, treatment, and outcomes in similar third-world countries.
\end{abstract}

Methods. Retrospective review of data of clubfoot patients seen at the Philippine General Hospital (PGH) Clubfoot Clinic from 2006 up to the present.

Results. Records from 75 patients treated at the PGH Clubfoot Clinic from 2010-2016 were reviewed. Idiopathic clubfoot comprised $76 \%$ of the patients, while syndromic clubfoot comprised $24 \%$. A good outcome of the Ponseti method was seen in $82 \%$ and $88 \%$ of the idiopathic and syndromic clubfoot patients, respectively. Idiopathic clubfoot cases that had good outcomes required an average of 11.84 casts to tenotomy or bracing, which was not statistically significant compared to 9.55 average sessions for syndromic clubfoot $(p=0.21)$. The initial Pirani scores for both cases were not significantly different $(p=0.95)$. Idiopathic cases with poor outcomes needed less casting sessions (4.45) because the decision to operate was made early. Age was not found to significantly affect the outcome of treatment for idiopathic clubfoot $(p=0.20)$ and syndromic clubfoot $(p=0.64)$.

Conclusion. Ponseti casting was found to be effective in treating both idiopathic and syndromic clubfoot patients. The number of sessions did not differ significantly between the two.

Key Words: Clubfoot, Congenital Talipes Equinovarus, Pediatric Orthopedics, Serial Casting, Ponseti Casting Method, Pirani Scoring

\section{INTRODUCTION}

Congenital Talipes Equinovarus (CTEV) is one of the most common congenital anomalies of the extremities, occurring in 1 in 1000 live births worldwide. ${ }^{1}$ The Global Clubfoot Initiative estimates the incidence in the Philippines to be $0.76 / 1000$ live births. ${ }^{1}$ When untreated, the burden of disease increases, mainly due to abnormalities with weightbearing and gait. Children with untreated clubfoot walk on the sides of their feet, causing callus formation, skin breakdown, potential sites of infection, impaired mobility, and limited employment opportunities..$^{2-5}$ The exact pathophysiology of CTEV remains controversial. However, numerous possible mechanisms have been described, from

${ }^{*}$ Dr. Eusebio and Dr. Sumpaico shared first authorship for this paper.

Corresponding author: Ilian Dominiq D. Eusebio, MD

Department of Orthopedics

Philippine General Hospital

University of the Philippines Manila

Taft Avenue, Ermita, Manila 1000, Philippines

Email: footandankle.ph@gmail.com genetic to environmental factors. ${ }^{2,5}$ Regardless of the cause, CTEV management has undergone radical changes in the past 50 years, shifting from early operative management to sequential non-surgical correction of deformities. ${ }^{3,5}$ Although CTEV treatment and outcomes have been extensively studied in other countries, there is no formal study yet of the condition here in the Philippines, and data is sparse regarding 
the epidemiology, treatment, and outcomes of this disease. This study aimed to describe the results of CTEV treatment using the Ponseti method at the Philippine General Hospital (PGH). As a tertiary and the national university hospital, this setting may be an adequate representation of health systems in place to treat CTEV in the country.

\section{Treatment Options}

Conservative measures are currently the standard of care for the management of CTEV. Options mainly revolve around the concept of gradual correction of deformities and immobilization to maintain the correction. ${ }^{6}$ Stretching is used in the French functional method, as well as Ponseti and Kite techniques. ${ }^{7}$ The Ponseti and Kite methods, however, use plaster casts to maintain reduction of the foot. The French method uses adhesive strips to maintain the correction. ${ }^{2}$ Botulinum toxin has also been used to relax the tight soft tissues surrounding the foot with modest success. ${ }^{8}$ Surgical options include Achilles tenotomy or tendon lengthening, tendon transfers, or gradual correction with an Ilizarov fixator. ${ }^{7}$ Surgery is often reserved for recalcitrant or relapsed cases, while Achilles tenotomy is usually done after the Ponseti method. ${ }^{2,3,5,7,9}$ Early surgical management is also associated with increased risk of developing arthritis in adulthood. ${ }^{10}$

The Ponseti method has emerged as the treatment of choice for CTEV worldwide. It is a specific manipulation method, reduction, casting, tenotomy, and bracing to achieve and maintain the correction. Cast changes are done every 5-7 days, and Achilles tenotomy is done if the dorsiflexion fails to improve beyond 15 degrees if the forefoot abduction is already 60-70 degrees. ${ }^{2,3}$ Uniformly good results have been obtained using this method even in underdeveloped countries., 3,5 Compared with the Kite method, the Ponseti method had similar results in deformity correction and maintenance of correction, fewer cast changes, greater reproducibility, and improvement in Pirani score. $3,5,711-13$ The disadvantage of the Ponseti method is the commitment to the treatment made both by the physician and the child's parents. Abduction bracing with specially designed orthoses are worn for 23 hours a day for the first three months, then at night for three years, making the total treatment time approximately four years. ${ }^{2,7}$ Intolerance to bracing is the primary reason for recurrence when the Ponseti method is used. ${ }^{2,3,14}$

\section{The Pirani Score and its Impact}

There is still no consensus on the grading of the severity of CTEV. ${ }^{4}$ Two of the most widely used systems are the Pirani and the Dimeglio scores. Both are based on clinical assessment of CTEV patients, and both have excellent inter and intraobserver reliability. 4,15 The predictive value of the Pirani scoring, however, remains controversial. Dyer and his colleagues did not find any linear relationship between the initial Pirani score and the total number of cast changes. ${ }^{8}$ These findings were also recorded by Gao and Chu. ${ }^{4,16}$ Despite these findings, the authors concluded that $92 \%$ of patients with an initial Pirani score $>4$ would need at least four casting sessions and that two components, posterior crease, and rigid equinus, had the highest correlation with the total number of casts required for correction ( $\mathrm{r} 0.09$ and 0.16). . $^{415,16}$ The Pirani score uniformly decreases with good outcomes of Ponseti casting. $4,5,9,16,17$

\section{Modifiers of Outcome}

The age of the patient upon presentation for treatment was found to have no correlation with treatment outcome. Successful treatment using the Ponseti method has been obtained in patients presenting at six months up to 9 years old. ${ }^{18,19}$ Iltar and colleagues, in a retrospective review, noted that Dimeglio scores were worse on final follow-up in patients treated before the $1^{\text {st }}$ month of life. ${ }^{13}$ They surmised that the difficulty lies in maintaining the reduction with plaster in a small foot. The non-ossified cuboid could not act as a barrier to compression, and cartilage remodeling needed to correct the supination.

Noh and Park suggested that the radiographic parameters measuring the tibiocalcaneal and lateral talocalcaneal angle correlate with the Pirani score after treatment. ${ }^{12}$ However, due to the unreliable radiographic findings in newborns and infants, these parameters have yet to be used for prognostication.

\section{Non-Idiopathic CTEV}

CTEV is also associated with several neuromuscular conditions. Of these, spine disorders (tethered cords, myelomeningocele), arthrogryposis, and cerebral palsy are most common. The neuromuscular disease has been associated with late relapses, and CTEV associated with arthrogryposis is described as "rigid" and "difficult to correct.", 20 Despite these difficulties, the Ponseti method is still being used as a first-line treatment for non-idiopathic CTE, and good outcomes have been reported..$^{621-25}$

\section{Barriers to Successful Treatment}

Management of CTEV in low and middle-income countries (LMIC) is challenging. Often, a health service delivery system has to be set up to provide care for these patients. Johnson and Friedman recognized this problem and conducted a systematic review. ${ }^{26}$ They classified barriers into three groups: 1) Patient-level - demographic and cultural variables that inhibit care, 2) Health system-level - supplies, personnel, or infrastructure, and 3) Provider-level - health care provider knowledge and training. Among all these factors, the most common cause for treatment failure is the patient level barriers; the negative stigma associated with CTEV and personal beliefs regarding the cause and treatment of the disease discourages patient consult and follow-up. The cost of treatment, including patient transportation and cost of materials, has also been found to negatively affect the treatment of CTEV in LMIC. This study included review articles from 24 LMIC, although the Philippines was not 
included since there is still no study describing the results of Ponseti treatment in our setting.

\section{METHODOLOGY}

\section{Study Design}

This study is a retrospective review of data of clubfoot patients seen at the PGH Clubfoot clinic from 2006 up to the present. Access to charts from the Clubfoot Clinic was done through the Medical Records Section of the PGH. Results of treatment of clubfoot were described for the three major groups of CTE: Idiopathic, Neurogenic, and Syndromic. Each group was compared with the other.

Residents of the Department of Orthopedics assessed patients at the Clubfoot Clinic of the PGH. The diagnosis was made with the supervision of consultants of the Pediatric Section of the Department. Following diagnosis, the patients were scored using the Pirani Score (Appendix, Image 1). Eligible patients were then treated according to the Ponseti Technique of serial casting (Appendix, Image 2). Subsequent follow-ups were also recorded until patients were shifted to the Denis-Brown abduction brace.

\section{Inclusion/Exclusion Criteria}

Patients seen at the PGH Clubfoot Clinic who have undergone serial casting using the Ponseti method were included in this study. Patients with positional clubfoot or metatarsus adductus were excluded from this study.

\section{Definition of Outcomes}

Outcomes were measured according to the type of procedure done upon completion of the Ponseti casting. These were grouped as follows: patients who underwent serial casting using the Ponseti technique with or without Achilles tenotomy or Achilles tendon lengthening (Group 1); and patients who underwent serial casting using the Ponseti technique and other procedures, including but not limited to posterior release, posteromedial release, lateral column shortening, or application of Ilizarov fixator (Group 2). The final position of the foot and the ambulation status of the patient were not included as an outcome measure.

\section{Data Handling}

Data were obtained from the PGH Clubfoot Clinic. Charts were accessed at the Medical Records Section of the PGH.

The database included the following information:

- Age at the first casting session

- Sex

- Laterality

- Diagnosis

- Pirani score

- Number of serial castings

- Treatment outcome

- Complications

\section{Statistical Analysis}

The STATA ${ }^{\text {TM }}$ data analysis and statistical software version 10 was used to analyze the data obtained. Categorical data (sex, diagnosis) are described in frequencies and percentages, continuous data (number of cast changes, age, Pirani score) are described using measures of central tendencies. Differences of means were measured using independent t-test. Correlations were tested using Spearman's coefficient. The significance level was set at a $\mathrm{p}$-value $<0.05$.

\section{Ethical Considerations}

All information was handled with strict confidentiality, with no patient-specific identifiers included in the final results of this paper. The study was a retrospective review of patient charts only. There was no contact with actual patients for this process. As such, there were neither risks nor benefits for the selected patients. Only the investigators had access to the data, and patient identity cannot be traced. All charts collected were not duplicated and were immediately returned to the PGH Records Section.

\section{RESULTS}

More than half $(56.8 \%)$ of the patients with clubfoot were male, and most (52.6\%) had bilateral foot involvement. About $61 \%$ of patients presented after one year of age. The majority (82\%) of patients had idiopathic clubfoot. $10 \%$ had neurogenic clubfoot, and $8 \%$ had syndromic clubfoot (Table 1, Figure 1).

Only 81 patients $(50.6 \%)$ had complete recording of treatment and outcomes. Analysis of data was therefore limited to this group of patients. Eighty-two (82\%) percent of patients with idiopathic clubfoot were included in Group 1. Similarly, $50 \%$ of neurogenic and $89 \%$ of syndromic patients were also included in Group 1 (Table 2, Figure 2). Compared to idiopathic clubfoot, outcomes of syndromic clubfoot and neurogenic were not significantly different $(p=0.76$ and 0.06 ).

Fifteen patients were included in Group 2. Nine patients needed additional surgical procedures to correct the deformity, while six patients had a relapse after Ponseti treatment (Table 3).

Table 4 shows the average number of Ponseti casting sessions needed for patients by outcome. Idiopathic clubfoot patients were successfully treated after an average of 11.8 casting sessions. In comparison, those who underwent additional procedures only had an average of 4.4 sessions. Neurogenic clubfoot required an average of 7 sessions for patients with a good outcome and 9.25 sessions for those requiring additional procedures. Patients with syndromic clubfoot treated with the classic Ponseti method required an average of 9.55 sessions, while those requiring additional procedures required 17 sessions. Applying the t-test, there was a significant difference between the mean number of casting sessions for idiopathic clubfoot patients depending on the outcome $(\mathrm{p}=0.0009)$ (Table 4, Figure 3$)$. 


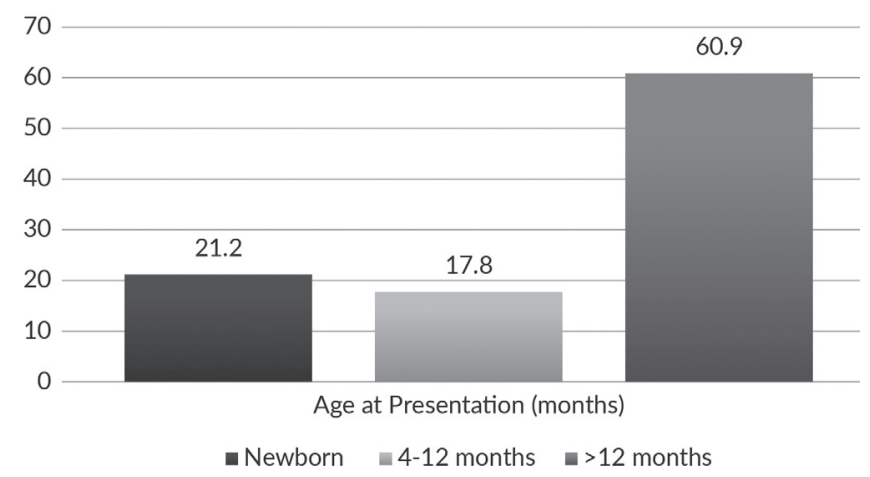

Figure 1. Age at presentation of patients.

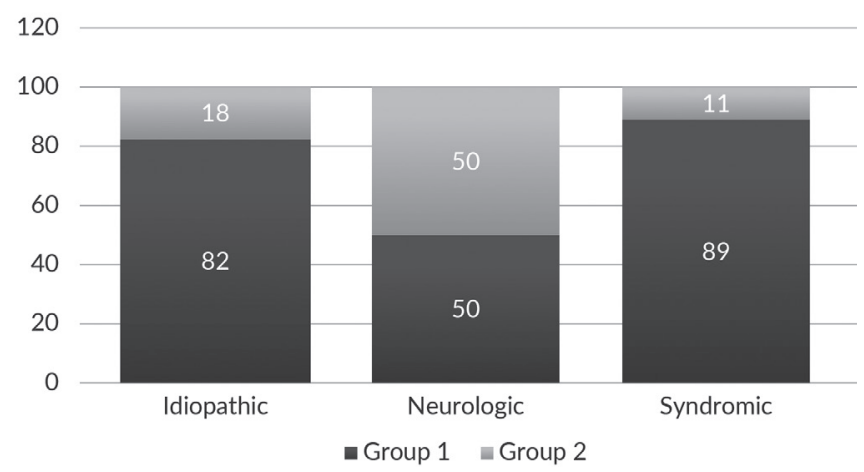

Figure 2. Classification of patients according to diagnosis and group.

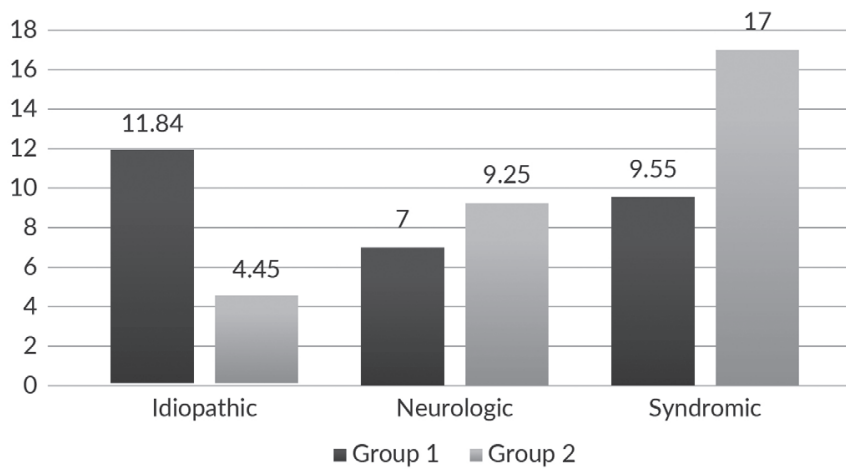

Figure 3. Average number of casting sessions per diagnosis.

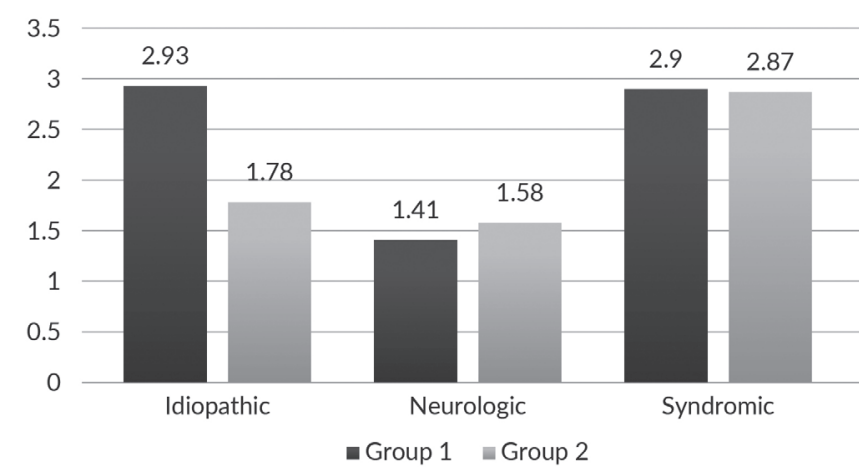

Figure 4. Mean initial Pirani Score.
Table 1. Patient Demographics

\begin{tabular}{ccclccc}
\multirow{2}{*}{ Diagnosis } & \multicolumn{2}{c}{ Sex } & & \multicolumn{3}{c}{ Laterality } \\
\cline { 2 - 5 } \cline { 5 - 6 } Idiopathic & $65(46.8)$ & $47(33.8)$ & & $28(20.7)$ & $25(18.5)$ & $56(41.5)$ \\
Neurogenic & $7(5.0)$ & $8(5.8)$ & & $6(4.4)$ & $1(0.7)$ & $7(5.2)$ \\
Syndromic & $7(5.0)$ & $5(3.6)$ & & $3(2.2)$ & $1(0.7)$ & $8(5.9)$ \\
\hline Total & $79(56.8)$ & $60(43.2)$ & $37(27.4)$ & $27(20.0)$ & $71(52.6)$ \\
\hline
\end{tabular}

Table 2. Outcomes of clubfoot patients

\begin{tabular}{ccccccc} 
& \multicolumn{7}{c}{ Outcomes } & \\
\cline { 2 - 6 } Assessment & Group 1 & $\%$ & Group 2 & $\%$ & Total & \\
Idiopathic & 47 & 82.46 & 10 & 17.54 & 57 & - \\
Neurogenic & 3 & 50.00 & 3 & 50.00 & 6 & 0.06 \\
Syndromic & 16 & 88.89 & 2 & 11.11 & 18 & 0.76 \\
\hline
\end{tabular}

Table 3. Breakdown of Group 2 outcomes

\begin{tabular}{ccc} 
Assessment & Relapsed & Surgically managed \\
\hline Idiopathic & 4 & 6 \\
Neurogenic & 1 & 2 \\
Syndromic & 1 & 1 \\
\hline
\end{tabular}

Table 4. Mean casting sessions per outcome

\begin{tabular}{|c|c|c|c|c|c|}
\hline \multirow{2}{*}{ Assessment } & \multicolumn{4}{|c|}{ Outcomes } & \multirow{2}{*}{ P-Value } \\
\hline & Group 1 & SD & Group 2 & SD & \\
\hline Idiopathic & 11.84 & 6.49 & 4.45 & 3.08 & 0.0009 \\
\hline Neurogenic & 7.00 & 2.05 & 9.25 & 0.47 & 0.1370 \\
\hline Syndromic & 9.55 & 5.50 & 17.00 & 9.00 & 0.0870 \\
\hline
\end{tabular}

Table 5. Mean initial Pirani score per outcome

\begin{tabular}{cccccc}
\multirow{2}{*}{ Assessment } & \multicolumn{5}{c}{ Outcomes } \\
\cline { 2 - 5 } & Group 1 & SD & Group 2 & SD & \\
\hline Idiopathic & 2.93 & 1.86 & 1.78 & 2.00 & 0.0852 \\
Neurogenic & 1.41 & 1.48 & 1.58 & 1.83 & 0.9065 \\
Syndromic & 2.90 & 1.68 & 2.87 & 2.63 & 0.9821 \\
\hline
\end{tabular}

The mean initial Pirani scores per type of clubfoot are shown in Table 5. The mean initial scores of patients in Group 1 were not significantly different from those included in Group 2 (Table 5, Figure 4).

\section{DISCUSSION}

The treatment of clubfoot was changed radically with the introduction of the Ponseti method. Although it is a safe, cost-effective method of treatment, clubfoot is still prevalent worldwide. A systematic review identified patient, health system, and health provider-level barriers to successful treatment. ${ }^{27}$ In our hospital, we encountered similar problems. Patient records were missing or incompletely filled, resulting in a decrease in the number of patients for 
analysis. These factors resulted in the exclusion of 85 patients from the analysis. However, in the past year, we have seen an increase in the number of patients enrolled in the Clubfoot Clinic. The increase in the number of patients seen at the clinic coincides with the increased effort of our Department to organize the record-keeping of clubfoot patients. Patientrelated factors may still affect the management of clubfoot in our setting - more than half of the patients still presented at our clinic at one year of age or older. Although our data echoes international literature that age at presentation does not seem to affect treatment outcome, this may represent the poor health-seeking behavior or the difficulty in accessing health care for our patients. Being a congenital deformity, clubfoot is easily recognized by parents and health care providers at birth or soon thereafter. As identified by Johnson, the social stigma of the disease and the patient's geographic location prevent them from seeking treatment immediately, leading to abnormal ambulation and all of its known sequelae. ${ }^{27}$

Despite these hindrances to treatment, our results parallel those in the current literature. The success rate of the Ponseti method was $82 \%$ for idiopathic cases, $89 \%$ for syndromic cases, and $50 \%$ for neurologic cases. These parallel the results in the current literature., $, 3,11,26$ Idiopathic clubfeet required more cast changes for Group 1 compared to those who underwent further surgical procedures. This may imply that recalcitrant cases are identified and scheduled for surgery early, or there might have been a misdiagnosis. However, from the current records, it was difficult to ascertain. The initial Pirani score of idiopathic cases also showed, paradoxically, higher scores for cases successfully treated. This was in contrast to the results shown by Dyer, who concluded that patients with an initial Pirani score of less than 4 required only 3 or 4 sessions. Agarwal and Gupta supported these results and showed a weak positive correlation $(r=0.05)$ between the initial Pirani score and the number of casts, with an increase in casting by 2 for every 3 point increase in Pirani score. ${ }^{8,28}$ In our series, the discrepancy in Pirani scoring might be due to inter and intraobserver variability in the scoring, as there was no designated scorer for the clinic. In contrast, nonidiopathic clubfeet failed to show an association between the Pirani score and outcome consistently.

Non-idiopathic clubfeet have been thought to be resistant to casting since they tend to be more rigid. In our study, patients with syndromic clubfeet required an average of about ten sessions for group 1 and 17 sessions for recalcitrant cases. Neurogenic cases, on the other hand, required 7 and 9.25 sessions, respectively. Both were found to be not significantly different, implying that the number of casting sessions done was not a good indicator of treatment success. Morcuende, in 2004 presented results of Ponseti casting for 16 patients with arthrogryposis, and for his series, 5-12 sessions were needed for correction, and five patients needed additional surgery. ${ }^{6}$ In 2009, Janicki published a comparative study on the outcomes of idiopathic and non-idiopathic clubfeet. ${ }^{23}$
Syndromic and neurogenic clubfeet were all grouped into non-idiopathic clubfeet. He reported a mean of 6 casting sessions for correction and a $10 \%$ failure rate for the Ponseti method. He also reported a recurrence rate of $44 \%$. Funk, in 2012, prospectively analyzed the outcomes of the Ponseti method for non-idiopathic clubfeet. ${ }^{25} \mathrm{He}$ also found that the Ponseti method successfully treated non-idiopathic clubfeet, even though the Pirani score was significantly higher in the non-idiopathic group. Our study's average initial Pirani score for syndromic cases was 2.9, and 1.41 for neurogenic cases included in Group 1. It was 2.97 and 1.58 respectively for those Group 2, and the values were not significantly different from each other. This may mean that the initial Pirani score was also not a good predictor for treatment success. It was well documented that the nonoperative management of clubfoot was successful in most cases.

This retrospective review showed higher rates of correction for idiopathic and syndromic cases compared with neurogenic cases. Significantly fewer casting sessions were needed for idiopathic clubfoot patients who underwent the modified Ponseti technique, indicating the increased suspicion for recalcitrant cases. Despite reports in the literature, the reliability of the initial Pirani score in prognostication was not shown in our study, especially for non-idiopathic clubfoot.

\section{CONCLUSION}

Poor health seeking behavior among parents and lack of access to health care may contribute to the severity of the disease at presentation and subsequent difficulty in treatment. Despite these hindrances, Ponseti casting was found to be effective in treating both idiopathic and syndromic clubfoot patients. The number of sessions required to correct the deformity did not differ significantly between the two. Neurogenic clubfoot presented as the least likely to be corrected with Ponseti casting. Patients with this kind of clubfoot should be counseled regarding the need for further treatment.

\section{Statement of Authorship}

Both authors participated in the data collection and analysis and approved the final version submitted.

\section{Author Disclosure}

Both authors declared no conflicts of interest.

\section{Funding Source}

No funding support.

\section{REFERENCES}

1. Global Clubfoot Initiative [Internet]. [cited 2017 June 19]. Available from: http://globalclubfoot.com/clubfoot/.

2. Dobbs MB, Gurnett, CA. Update on Clubfoot: Etiology and Treatment. Clin Orthop Relat Res 467 (2009): 1146-1153. doi: 10.1007/s11999009-0734-9. 
3. Radler C. The Ponseti Method for the Treatment of Congenital Clubfoot: Review of the Current Literature and Treatment Recommendations. Int Orthop 37 (2013): 1747-1753. doi: 10.1007/ s00264-013-2031-1.

4. Gao R, Tomlimson M, Walker C. Correlation of Pirani and Dimeglio Scores with Number of Ponseti Casts Required for Clubfoot Correction. J Pediatr Orthop 34 (2014): 639-642. doi: 10.1097/ BPO.0000000000000182.

5. Jowett CR, Morcuende JA and Ramachandran M. Management of Congenital Talipes Equinovarus Using the Ponseti Method: A systematic Review. J Bone Joint Surg Br 93-B (2011): 1160-1164. doi: 10.1302/0301-620X.93B9.26947.

6. Morcuende JA, Dobbs MB, Frick SL. Results of Ponseti Method in Patients with Clubfoot Associated with Arthrogryposis. Iowa Orthop J 23. 2008; 28: 22-6. PMID: 19223944; PMCID: PMC2603345.

7. Gray K. Interventions for Congenital Talipes Equinovarus (Clubfoot) (Review). Cochrane Database Syst Rev. 2014 Aug 12;2014(8):CD008602. doi: 10.1002/14651858.CD008602.pub3.

8. Richards BS, Faulks S, Rathjen KE, Karol LA, Johnston CB, Jones SA. A Comparison of Two Nonoperative Methods of Idiopathic Clubfoot Correction: The Ponseti Method and the French Functional (Physiotherapy) Method. J Bone Joint Surg Am 2008 Nov;90(11):2313-21. doi: 10.2106/JBJS.G.01621.

9. Alvarez CM, Tredwell SJ, Keenan SP, Beauchamp RD, Choit RL, Sawatzky BJ, et al. Treatment of Idiopathic Clubfoot Utilizing Botulinum A Toxin: A New Method and Its Short-term Outcomes. J Pediatr Orthop 2005;25(2):229-35.

10. Dobbs MB, Nunley R, Schoenecker PL. Long-term Follow-up of Patients with Clubfeet Treated with Extensive Soft-tissue Release. J Bone Joint Surg Am. 2006;88:986-96.

11. Dyer PJ, Davis N. The Role of the Pirani Scoring System in the Management of Clubfoot by the Ponseti Method. J Bone Joint Surg Br 2006 Aug;88(8):1082-4. doi: 10.1302/0301-620X.88B8.17482.

12. Noh H, Park SS. Predictive Factors for Residual Equinovarus Deformity Follow ing Ponseti Treatment and Percutaneous Achilles Tenotomy for Idiopathic Clubfoot: A Retrospective Review of 50 Cases Followed for Median 2 Years. Acta Orthop 2013 Apr; 84(2): 213-7. doi: 10.3109/17453674.2013.784659.

13. Iltar S, Usyal M, Alemdaroglu KB, Aydogan NH, Kara T, Atlihan D. Treatment of Clubfoot with the Ponseti Method: Should We Begin Casting in the Newborn Period or Later?. J Foot Ankle Surg. Sep-Oct 2010;49(5):426-31. doi: 10.1053/j.jfas.2010.06.010.

14. Dobbs MB, Rudzki JR, Purcell DB, Walton T, Porter KR, Gurnett CA. Factors Predictive of Outcome After Use of the Ponseti Method for the Treatment of Idiopathic Clubfeet. J Bone Joint Surg Am. 2004 Jan;86(1):22-7. doi: 10.2106/00004623-200401000-00005.

15. Flynn JM, Donohoe M, Mackenzie WG. An Independent Assessment of Two Clubfoot-classification Systems. J Pediatr Orthop. May-Jun 1998;18(3):323-7.

16. Chu A, Labar AS, Sala DA, van Bosse HJP, Lehman W. Clubfoot Classification: Correlation with Ponseti Cast Treatment. J Pediatr Orthop. Oct-Nov 2010;30(7):695-9. doi: 10.1097/BPO. 0b013e3181ec0853.
17. Sud A, Tiwari A, Sharma D, Kapoor S. Ponseti's vs. Kite's Method in the Treatment of Clubfoot: A Prospective Randomised Study. Int Orthop 2008 Jun; 32(3): 409-13.

18. Sanghvi AV, Mittal VK. Conservative Management of Idiopathic Clubfoot: Kite versus Ponseti Method. J Orthop Surg (Hong Kong). 2009 Apr;17(1):67-71.

19. Rijal R, Shrestha BP, Singh GK, Singh M, Nepal P, Khanal GP, et al. Comparison of Ponseti and Kite's Method of Treatment for Idiopathic Clubfoot. Indian J Orthop 2010 Apr;44(2):202-7. doi: 10.4103/00195413.61941.

20. Alves C, Escalda C, Fernandes P, Tavares D, Neves MC. Ponseti Method: Does Age at the Beginning of Treatment Make a Difference? Clin Orthop Relat Res. 2009 May; 467(5): 1271-7. doi: 10.1007/ s11999-008-0698-1.

21. Lourenço AF, Morcuende JA. Correction of Neglected Idiopathic Clubfoot by the Ponseti Method. J Bone Joint Surg Br. 2007 Mar;89(3):378-81. doi: 10.1302/0301-620X.89B3.18313.

22. Lovell ME, Morcuende JA. Neuromuscular Disease as the Cause of Late Relapses: Report of 4 Cases. Iowa Orthop J 2007;27:82-84.

23. Janicki JA, Narayanan UG, Harvey B, Roy A, Ramseier LE, Wright JG. Treatment of Neuromuscular and Syndrome-associated (Non-idiopathic) Clubfeet Using the Ponseti Method. J Pediatr Orthop. 2007;27:82-4.2009 Jun;29(4):393-7. doi: 10.1097/BPO. 0b013e3181a6bf77.

24. Matar HE, Bierne P, Bruce CE, Garg NK. Treatment of Complex Idiopathic Clubfoot Using the Modified Ponseti Method: Up to 11 Years Follow-up. J Pediatr Orthop B 2017 Mar;26(2):137-42. doi: 10.1097/BPB.0000000000000321.

25. Funk JF, Lebek S, Seidl T, Placzek R. Comparison of Treatment Results of Idiopathic and Non-idiopathic Congenital Clubfoot. Prospective Evaluation of the Ponseti Therapy. Orthopade. 2012 Dec;41(12): 977-83. doi: 10.1007/s00132-012-1982-z.

26. Ippolito E, Farsetti P, Caterini R, Tudisco C. Long-term Comparative Results in Patients With Congenital Clubfoot Treated with Two Different Protocols. J Bone Joint Surg Am. 2003 Jul;85(7):1286-94. doi: 10.2106/00004623-200307000-00015.

27. Johnson RR, Friedman JM, Becker AM, Spiegel DA. The Ponseti Method for Clubfoot Treatment in Low and Middle-Income Countries: A Systematic Review of Barriers and Solutions to Service Delivery. J Pediatr Orthop. 2017 Mar;37(2):e134-e139. doi: 10.1097/ BPO.0000000000000723. PMID: 26919713.

28. Agarwal A. Gupta N. Does Initial Pirani Score Influence the Number of Ponseti Casts in Children?. Int Orthop 2014 Mar; 38(3): 569-72. doi: 10.1007/s00264-013-2155-3.

29. Pirani S, Staheli L, Naddumba E. Ponseti Clubfoot Management: Teaching Manual for Health-care Providers in Uganda. Manual. 2008. GHO Publications. [Internet]. [cited 2017 June 19]. Available from: $<$ https://global-help.org/products/ponseti_clubfoot_management_ teaching_manual_for_health-care_providers_in_uganda/>

30. Ponseti Checklist. [Internet]. [cited 2017 June 19]. Available from: $<$ https://clubfootclub.org/about/ponseti-checklist/> 


\section{APPENDICES}
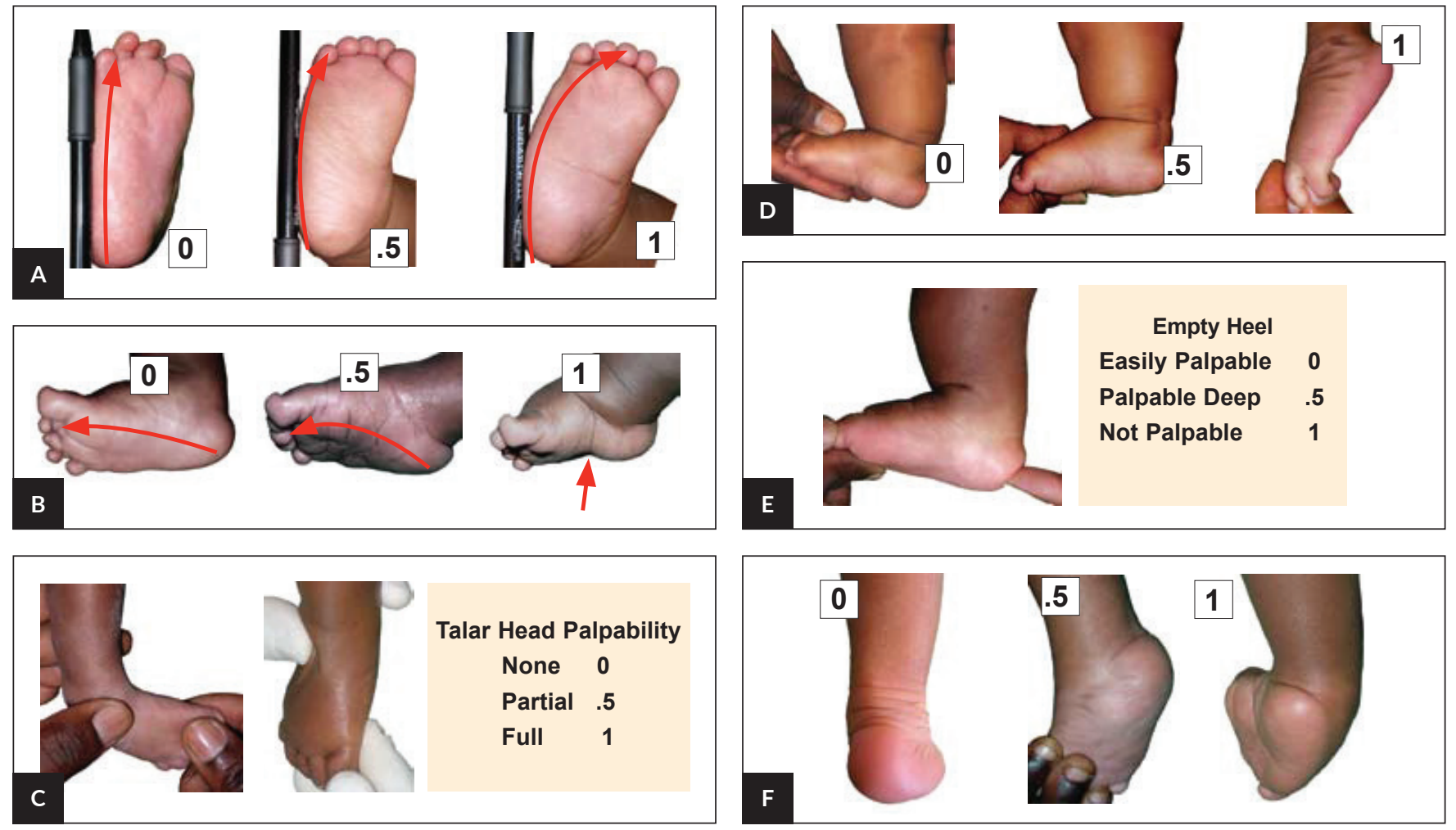

Appendix Figure 1. The Pirani Scoring System. ${ }^{29}$ (A) Curved lateral border, (B) Medial fold (cavus), (C) Palpation of the talar head, (D) Rigid equinus, (E) Palpation of calcaneus, (F) Posterior fold.

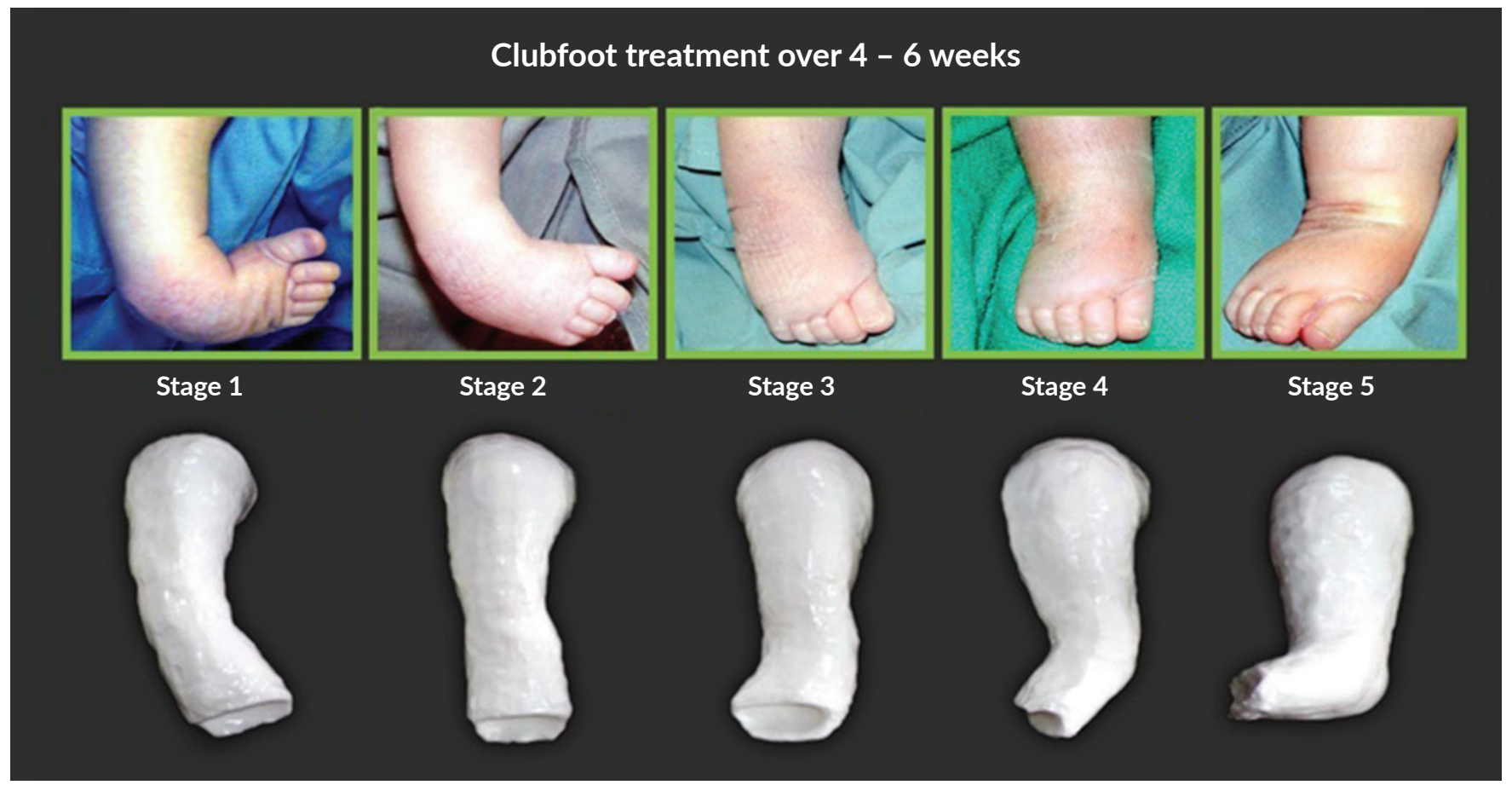

Appendix Figure 2. Ponseti Casting Technique. ${ }^{30}$ 